Acta Crystallographica Section E

Structure Reports

Online

ISSN 1600-5368

\section{1-\{[(2,3-Dihydro-1H-inden-2-yl)oxy]- methyl\}quinazoline-2,4(1H,3H)-dione}

\author{
Nasser R. El-Brollosy, ${ }^{a}$ Necmi Dege, ${ }^{b}$ Güneș Demirtaș, ${ }^{\text {b* }}$ \\ Mohamed I. Attia, ${ }^{a}$ Ali A. El-Emam ${ }^{a}$ and Orhan \\ Büyükgüngör ${ }^{b}$
}

${ }^{a}$ Department of Pharmaceutical Chemistry, College of Pharmacy, King Saud University, 11451 Riyadh, Saudi Arabia, and ${ }^{\mathbf{b}}$ Department of Physics, Faculty of Arts and Sciences, Ondokuz Mayıs University, 55139 Samsun, Turkey

Correspondence e-mail: gunesd@omu.edu.tr

Received 26 April 2012; accepted 16 May 2012

Key indicators: single-crystal X-ray study; $T=296 \mathrm{~K}$; mean $\sigma(\mathrm{C}-\mathrm{C})=0.003 \AA$; $R$ factor $=0.044 ; w R$ factor $=0.103 ;$ data-to-parameter ratio $=15.2$.

In the title molecule, $\mathrm{C}_{18} \mathrm{H}_{16} \mathrm{~N}_{2} \mathrm{O}_{3}$, the five-membered ring has an envelope conformation, with the substituted $\mathrm{C}$ atom deviating by 0.342 (4) $\AA$ from the mean plane $P$ calculated for the remainder of the non-H atoms of the 2,3-dihydro- $1 \mathrm{H}$ indene fragment. The mean planes of quinazoline-2,4(1H,3H)dione fragment and $P$ form a dihedral angle of $59.08(4)^{\circ}$. In the crystal, pairs of $\mathrm{N}-\mathrm{H} \cdots \mathrm{O}$ hydrogen bonds link molecules into inversion dimers, and weak $\mathrm{C}-\mathrm{H} \cdots \mathrm{O}$ hydrogen bonds and $\pi-\pi$ interactions between the benzene rings of the quinazoline ring systems [centroid-centroid distance = 3.538 (3) §] further consolidate the packing.

\section{Related literature}

For the biological activity of quinazoline-2,4(1H,3H)-diones, see: Tran et al. (2004); Cao et al. (2010) and for the biological activity of non-nucleoside reverse transcriptase inhibitors (NNRTIs), see: Hopkins et al. (1996, 1999); El-Brollosy (2006, 2007); El-Brollosy et al. (2008, 2009). For related structures, see: Liu (2008); Karimova et al. (2010).

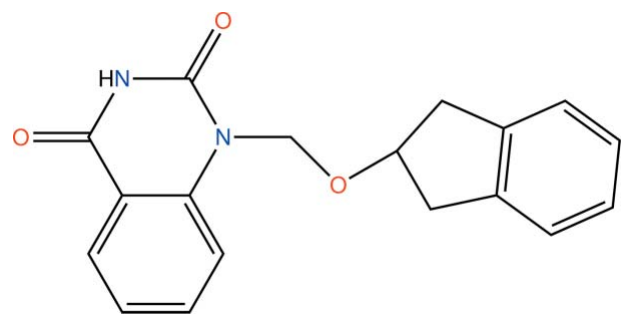

\section{Experimental}

Crystal data

$\mathrm{C}_{18} \mathrm{H}_{16} \mathrm{~N}_{2} \mathrm{O}_{3}$

$M_{r}=308.33$

Triclinic, $P \overline{1}$ $\alpha=87.199(8)^{\circ}$
$\beta=78.332(8)^{\circ}$
$\gamma=70.569(8)^{\circ}$
$V=761.28(13) \AA^{3}$
$Z=2$

Data collection

Stoe IPDS 2 diffractometer

Absorption correction: integration (X-RED32; Stoe \& Cie, 2002)

$T_{\min }=0.948, T_{\max }=0.995$

Refinement

$R\left[F^{2}>2 \sigma\left(F^{2}\right)\right]=0.044$

$w R\left(F^{2}\right)=0.103$

$S=1.00$

3156 reflections
Mo $K \alpha$ radiation

$\mu=0.09 \mathrm{~mm}^{-1}$

$T=296 \mathrm{~K}$

$0.58 \times 0.38 \times 0.05 \mathrm{~mm}$

Table 1

Hydrogen-bond geometry $\left(\AA{ }^{\circ}\right)$.

\begin{tabular}{lllll}
\hline$D-\mathrm{H} \cdots A$ & $D-\mathrm{H}$ & $\mathrm{H} \cdots A$ & $D \cdots A$ & $D-\mathrm{H} \cdots A$ \\
\hline $\mathrm{N} 2-\mathrm{H} 2 \cdots \mathrm{O} 2^{\mathrm{i}}$ & 0.86 & 2.06 & $2.9106(18)$ & 169 \\
$\mathrm{C} 9-\mathrm{H} 9 A \cdots \mathrm{O} 2^{\mathrm{ii}}$ & 0.97 & 2.56 & $3.527(3)$ & 173 \\
$\mathrm{C} 16-\mathrm{H} 16 \cdots{ }^{\mathrm{iii}}$ & 0.93 & 2.47 & $3.378(2)$ & 166 \\
$\mathrm{C} 10-\mathrm{H} 10 A \cdots \mathrm{O}^{\mathrm{iv}}$ & 0.97 & 2.46 & $3.404(2)$ & 165 \\
$\mathrm{C} 5-\mathrm{H} 5 \cdots \mathrm{O}^{\mathrm{v}}$ & 0.93 & 2.47 & $3.314(2)$ & 151 \\
\hline Symmetry codes: & (i) $-x,-y+1,-z ;$ & (ii) & $-x+1,-y+1,-z ;$ & (iii) \\
$-x+1,-y+1,-z+1 ;$ (iv) $x+1, y, z ;$ (v) $x+1, y+1, z$. &
\end{tabular}

Data collection: $X$-AREA (Stoe \& Cie, 2002); cell refinement: $X$ $A R E A$; data reduction: $X$-RED32 (Stoe \& Cie, 2002); program(s) used to solve structure: WinGX (Farrugia, 1997) and SHELXS97 (Sheldrick, 2008); program(s) used to refine structure: SHELXL97 (Sheldrick, 2008); molecular graphics: ORTEP-3 for Windows (Farrugia, 1997); software used to prepare material for publication: WinGX (Farrugia, 1999) and PLATON (Spek, 2009).

The authors thank the Ondokuz Mayıs University Research Fund for financial support. The financial support of the Deanship of Scientific Research and the Research Center of the College of Pharmacy, King Saud University, is greatly appreciated.

Supplementary data and figures for this paper are available from the IUCr electronic archives (Reference: CV5291).

\title{
References
}

Cao, S.-L., Wang, Y., Zhu, L., Liao, J., Guo, Y.-W., Chen, L.-L., Liu, H.-Q. \& Xu, X. (2010). Eur. J. Med. Chem. 45, 3850-3857.

El-Brollosy, N. R. (2006). J. Heterocycl. Chem. 43, 1435-1440.

El-Brollosy, N. R. (2007). J. Chem. Res. pp. 358-361.

El-Brollosy, N. R., Al-Deeb, O. A., El-Emam, A. A., Pedersen, E. B., La Colla, P., Collu, G., Sanna, G. \& Loddo, R. (2009). Arch. Pharm. Chem. Life Sci. 342, 663-670.

El-Brollosy, N. R., Sorensen, E. R., Pedersen, E. B., Sanna, G., La Colla, P. \& Loddo, R. (2008). Arch. Pharm. Chem. Life Sci. 341, 9-19.

Farrugia, L. J. (1997). J. Appl. Cryst. 30, 565.

Farrugia, L. J. (1999). J. Appl. Cryst. 32, 837-838.

Hopkins, A. L., Ren, J., Esnouf, R. M., Willcox, B. E., Jones, E. Y., Ross, C., Miyasaka, T., Walker, R. T., Tanaka, H., Stammers, D. K. \& Stuart, D. I. (1996). J. Med. Chem. 39, 1589-1600.

Hopkins, A. L., Ren, J., Tanaka, H., Baba, M., Okamato, M., Stuart, D. I. \& Stammers, D. K. (1999). J. Med. Chem. 42, 4500-4505. 


\section{organic compounds}

Karimova, G., Ashurov, J., Mukhamedov, N., Parpiev, N. A. \& Shakhidoyatov, K. M. (2010). Acta Cryst. E66, o1547.

Liu, G. (2008). Acta Cryst. E64, o1677.

Sheldrick, G. M. (2008). Acta Cryst. A64, 112-122.

Spek, A. L. (2009). Acta Cryst. D65, 148-155.
Stoe \& Cie (2002). $X$ - $A R E A$ and $X$-RED32. Stoe \& Cie, Darmstadt, Germany. Tran, T. P., Ellsworth, E. L., Stier, M. A., Domagala, J. M., Showalter, H. D., Gracheck, S. J., Shapiro, M. A., Joannides, T. E. \& Singh, R. (2004). Bioorg. Med. Chem. Lett. 14, 4405-4409. 


\section{supporting information}

Acta Cryst. (2012). E68, o1866-o1867 [doi:10.1107/S1600536812022350]

\section{1-\{[(2,3-Dihydro-1H-inden-2-yl)oxy]methyl\}quinazoline-2,4(1H,3H)-dione \\ Nasser R. El-Brollosy, Necmi Dege, Güneş Demirtaş, Mohamed I. Attia, Ali A. El-Emam and Orhan Büyükgüngör}

\section{S1. Comment}

Non-nucleoside reverse transcriptase inhibitors (NNRTIs) are very promising therapies in the treatment of human immunodeficiency virus (HIV) (Hopkins et al., 1996, 1999). Some series of 3-hydroxyquinazoline-2,4-dione and $N$-((2methyl-4(3H)-quinazolinon-6-yl)methyl)dithiocarbamates have been synthesized and evulated for antibacterial activity (Tran et al. 2004; Cao et al. 2010). In continuation to our interest in NNRTIs (El-Brollosy et al. 2006, 2007, 2008, 2009), we synthesized the title compound, (I), as a potential non-nucleoside reverse transcriptase inhibitor.

In (I) (Fig. 1), in the 2,3-dihydro- $1 H$-indene fragment atom C1deviates from the main plane $P$ at 0.342 (4) $\AA$. In the literature, some quinazoline-2,4(1H,3H)-dione structures have been reported (Liu, 2008; Karimova et al. 2010). The $\mathrm{C} 11=\mathrm{O} 2$ and $\mathrm{C} 12=\mathrm{O} 3$ bond lengths are 1.2247 (19) and 1.2144 (18) $\AA$, respectively. The $\mathrm{C} \cdots \mathrm{C}$ bond distances range from 1.362 (3) $\AA$ to1.394 (2) $\AA$. The torsion angle $\mathrm{C} 1-\mathrm{O} 1-\mathrm{C} 10-\mathrm{N} 1$ is -93.88 (17).

In the crystal, intermolecular $\mathrm{N} 2-\mathrm{H} 2 \cdots \mathrm{O} 2$ hydrogen bond (Table 1) link two molecules into centrosymmetric dimer. Further, weak $\mathrm{C}-\mathrm{H} \cdots \mathrm{O}$ hydrogen bonds (Table 1 ) and $\pi-\pi$ interactions between the benzene rings of the quinazoline bicycles [centroid-centroid distance $=3.538$ (3) $\AA$ ] consolidate the crystal packing.

\section{S2. Experimental}

Quinazoline-2,4(1H,3H)-dione (162 mg, $1 \mathrm{mmol})$ was stirred in dry acetonitrile $(15 \mathrm{ml})$ under nitrogen and N,O-bis(trimethylsilyl)acetamide (BSA) $(0.87 \mathrm{ml}, 3.5 \mathrm{mmol})$ was added. After a clear solution was obtained $(10 \mathrm{~min})$, the mixture was cooled down to $-50{ }^{\circ} \mathrm{C}$ and $T M S$ trifluoromethanesulfonate $(0.18 \mathrm{ml}, 1 \mathrm{mmol})$ was added followed by the dropwise addition of bis(indan-2-yloxy)methane $(560 \mathrm{~g}, 2 \mathrm{mmol})$. The reaction mixture was stirred at room temperature for $5 \mathrm{~h}$, and quenched by addition of saturated aqueous sodium hydrogen carbonate solution $(5 \mathrm{ml})$. The mixture was evaporated under reduced pressure and the residue was extracted with ether $(3 \times 50 \mathrm{ml})$. The combined ether fractions were dried $\left(\mathrm{MgSO}_{4}\right)$ and evaporated under reduced pressure. The product was purified on silica gel column chromatography, using $20 \%$ ether in petroleum ether $\left(40-60^{\circ} \mathrm{C}\right)$, to afford the title compound as a white solid in $71 \%$ yield $(218 \mathrm{mg})$. Single crystals were achieved by crystallization from ethanol. M.p. 193-194 ${ }^{\circ} \mathrm{C}$ (El-Brollosy, 2007).

\section{S3. Refinement}

All $\mathrm{H}$ atoms were positioned geometrically $[\mathrm{N}-\mathrm{H}=0.86 \AA ; \mathrm{C}-\mathrm{H}=0.93 \AA-0.98 \AA]$ and treated as riding, with $U_{\text {iso }}(\mathrm{H})=1.2 U_{\mathrm{eq}}(\mathrm{C}, \mathrm{N})$. 


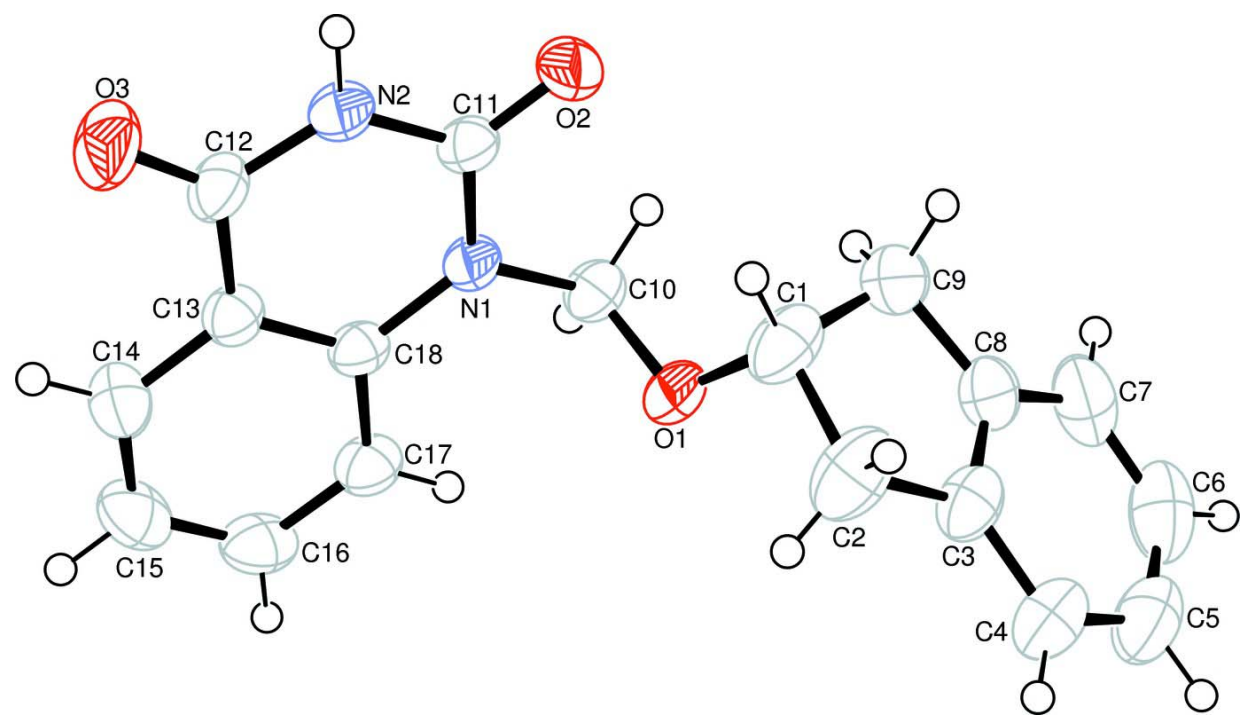

\section{Figure 1}

The molecular structure of (I) showing the atomic numbering scheme. Displacement ellipsoids are drawn at the $50 \%$ probability level.

\section{1-\{[(2,3-Dihydro-1 $H$-inden-2-yl)oxy]methyl\}quinazoline- 2,4(1H,3H)-dione}

\section{Crystal data}

$\mathrm{C}_{18} \mathrm{H}_{16} \mathrm{~N}_{2} \mathrm{O}_{3}$

$M_{r}=308.33$

Triclinic, $P \overline{1}$

Hall symbol: -P 1

$a=7.6684(8) \AA$

$b=10.0717(10) \AA$

$c=10.6748(11) \AA$

$\alpha=87.199(8)^{\circ}$

$\beta=78.332(8)^{\circ}$

$\gamma=70.569(8)^{\circ}$

$V=761.28(13) \AA^{3}$

Data collection

Stoe IPDS 2

diffractometer

Radiation source: fine-focus sealed tube

Graphite monochromator

rotation method scans

Absorption correction: integration

(X-RED32; Stoe \& Cie, 2002)

$T_{\min }=0.948, T_{\max }=0.995$

Refinement

Refinement on $F^{2}$

Least-squares matrix: full

$R\left[F^{2}>2 \sigma\left(F^{2}\right)\right]=0.044$

$w R\left(F^{2}\right)=0.103$

$S=1.00$

3156 reflections
$Z=2$

$F(000)=324$

$D_{\mathrm{x}}=1.345 \mathrm{Mg} \mathrm{m}^{-3}$

Mo $K \alpha$ radiation, $\lambda=0.71073 \AA$

Cell parameters from 11963 reflections

$\theta=2.9-27.9^{\circ}$

$\mu=0.09 \mathrm{~mm}^{-1}$

$T=296 \mathrm{~K}$

Plate, colorless

$0.58 \times 0.38 \times 0.05 \mathrm{~mm}$

11601 measured reflections

3156 independent reflections

2078 reflections with $I>2 \sigma(I)$

$R_{\text {int }}=0.043$

$\theta_{\max }=26.5^{\circ}, \theta_{\min }=2.9^{\circ}$

$h=-9 \rightarrow 9$

$k=-12 \rightarrow 12$

$l=-13 \rightarrow 13$

\section{8 parameters}

0 restraints

Primary atom site location: structure-invariant direct methods

Secondary atom site location: difference Fourier map 
Hydrogen site location: inferred from neighbouring sites

H-atom parameters constrained

$$
\begin{gathered}
w=1 /\left[\sigma^{2}\left(F_{\mathrm{o}}{ }^{2}\right)+(0.0506 P)^{2}\right] \\
\text { where } P=\left(F_{\mathrm{o}}^{2}+2 F_{\mathrm{c}}{ }^{2}\right) / 3 \\
(\Delta / \sigma)_{\max }<0.001 \\
\Delta \rho_{\max }=0.15 \mathrm{e} \AA^{-3} \\
\Delta \rho_{\min }=-0.16 \mathrm{e} \AA^{-3}
\end{gathered}
$$

Special details

Geometry. All e.s.d.'s (except the e.s.d. in the dihedral angle between two 1.s. planes) are estimated using the full covariance matrix. The cell e.s.d.'s are taken into account individually in the estimation of e.s.d.'s in distances, angles and torsion angles; correlations between e.s.d.'s in cell parameters are only used when they are defined by crystal symmetry. An approximate (isotropic) treatment of cell e.s.d.'s is used for estimating e.s.d.'s involving 1.s. planes.

Refinement. Refinement of $F^{2}$ against ALL reflections. The weighted $R$-factor $w R$ and goodness of fit $S$ are based on $F^{2}$, conventional $R$-factors $R$ are based on $F$, with $F$ set to zero for negative $F^{2}$. The threshold expression of $F^{2}>\sigma\left(F^{2}\right)$ is used only for calculating $R$-factors(gt) etc. and is not relevant to the choice of reflections for refinement. $R$-factors based on $F^{2}$ are statistically about twice as large as those based on $F$, and $R$ - factors based on ALL data will be even larger.

Fractional atomic coordinates and isotropic or equivalent isotropic displacement parameters $\left(\AA^{2}\right)$

\begin{tabular}{lllll}
\hline & $x$ & $y$ & $z$ & $U_{\text {iso }} / U_{\text {eq }}$ \\
\hline C1 & $0.3360(3)$ & $0.7691(2)$ & $0.1809(2)$ & $0.0586(5)$ \\
H1 & 0.2262 & 0.7682 & 0.1477 & $0.070^{*}$ \\
C2 & $0.2880(3)$ & $0.8977(2)$ & $0.2672(3)$ & $0.0688(6)$ \\
H2A & 0.1797 & 0.9729 & 0.2480 & $0.083^{*}$ \\
H2B & 0.2613 & 0.8741 & 0.3566 & $0.083^{*}$ \\
C3 & $0.4616(3)$ & $0.94003(18)$ & $0.23750(19)$ & $0.0500(5)$ \\
C4 & $0.5098(3)$ & $1.0335(2)$ & $0.3022(2)$ & $0.0609(5)$ \\
H4 & 0.4299 & 1.0798 & 0.3760 & $0.073^{*}$ \\
C5 & $0.6771(3)$ & $1.0575(2)$ & $0.2565(3)$ & $0.0705(6)$ \\
H5 & 0.7098 & 1.1214 & 0.2990 & $0.085^{*}$ \\
C6 & $0.7955(3)$ & $0.9884(3)$ & $0.1493(3)$ & $0.0755(7)$ \\
H6 & 0.9087 & 1.0055 & 0.1198 & $0.091^{*}$ \\
C7 & $0.7506(3)$ & $0.8938(2)$ & $0.0837(2)$ & $0.0715(6)$ \\
H7 & 0.8329 & 0.8462 & 0.0112 & $0.086^{*}$ \\
C8 & $0.5807(3)$ & $0.87095(18)$ & $0.12792(19)$ & $0.0529(5)$ \\
C9 & $0.4947(3)$ & $0.7796(2)$ & $0.07299(19)$ & $0.0657(6)$ \\
H9A & 0.5867 & 0.6874 & 0.0489 & $0.079^{*}$ \\
H9B & 0.4456 & 0.8222 & -0.0017 & $0.079^{*}$ \\
C10 & $0.4294(2)$ & $0.51886(17)$ & $0.19832(17)$ & $0.0416(4)$ \\
H10A & 0.5368 & 0.4475 & 0.2233 & $0.050^{*}$ \\
H10B & 0.4588 & 0.5283 & 0.1063 & $0.050^{*}$ \\
C11 & $0.1516(2)$ & $0.48967(16)$ & $0.14342(15)$ & $0.0366(4)$ \\
C12 & $-0.0368(2)$ & $0.36456(17)$ & $0.28236(17)$ & $0.0422(4)$ \\
C13 & $0.0822(2)$ & $0.35258(16)$ & $0.37605(15)$ & $0.0379(4)$ \\
C14 & $0.0470(3)$ & $0.28777(19)$ & $0.49221(17)$ & $0.0495(4)$ \\
H14 & -0.0524 & 0.2519 & 0.5098 & $0.059^{*}$ \\
C15 & $0.1577(3)$ & $0.27652(19)$ & $0.58079(18)$ & $0.0548(5)$ \\
H15 & 0.1350 & 0.2321 & 0.6579 & $0.066^{*}$ \\
C16 & $0.3030(3)$ & $0.33168(19)$ & $0.55437(17)$ & $0.0515(5)$ \\
H16 & 0.3775 & 0.3247 & 0.6148 & $0.062^{*}$ \\
& & & &
\end{tabular}


supporting information

$\begin{array}{lllll}\mathrm{C} 17 & 0.3401(2) & 0.39664(18) & 0.44111(16) & 0.0446(4) \\ \mathrm{H} 17 & 0.4386 & 0.4335 & 0.4253 & 0.053^{*} \\ \mathrm{C} 18 & 0.2296(2) & 0.40725(16) & 0.34981(15) & 0.0350(4) \\ \mathrm{N} 1 & 0.26417(18) & 0.47265(13) & 0.23194(12) & 0.0356(3) \\ \mathrm{N} 2 & 0.00901(19) & 0.43362(14) & 0.17284(13) & 0.0422(3) \\ \mathrm{H} 2 & -0.0598 & 0.4425 & 0.1165 & 0.051^{*} \\ \mathrm{O} 1 & 0.40148(17) & 0.64742(12) & 0.25756(11) & 0.0487(3) \\ \mathrm{O} 2 & 0.17725(17) & 0.54975(13) & 0.04228(11) & 0.0490(3) \\ \mathrm{O} 3 & -0.16859(19) & 0.32033(15) & 0.29619(14) & 0.0658(4)\end{array}$

Atomic displacement parameters $\left(\AA^{2}\right)$

\begin{tabular}{lllllll}
\hline & $U^{11}$ & $U^{22}$ & $U^{33}$ & $U^{12}$ & $U^{13}$ & $U^{23}$ \\
\hline C1 & $0.0577(12)$ & $0.0473(10)$ & $0.0821(15)$ & $-0.0195(9)$ & $-0.0355(11)$ & $0.0023(10)$ \\
C2 & $0.0494(11)$ & $0.0560(12)$ & $0.1013(18)$ & $-0.0166(10)$ & $-0.0134(11)$ & $-0.0133(11)$ \\
C3 & $0.0489(10)$ & $0.0378(9)$ & $0.0665(13)$ & $-0.0140(8)$ & $-0.0192(9)$ & $0.0032(9)$ \\
C4 & $0.0620(12)$ & $0.0467(11)$ & $0.0785(15)$ & $-0.0201(9)$ & $-0.0190(11)$ & $-0.0048(10)$ \\
C5 & $0.0794(16)$ & $0.0567(12)$ & $0.0951(18)$ & $-0.0362(12)$ & $-0.0405(14)$ & $0.0135(12)$ \\
C6 & $0.0627(14)$ & $0.0710(15)$ & $0.102(2)$ & $-0.0354(12)$ & $-0.0199(14)$ & $0.0302(14)$ \\
C7 & $0.0780(15)$ & $0.0614(13)$ & $0.0697(15)$ & $-0.0267(12)$ & $-0.0002(12)$ & $0.0189(11)$ \\
C8 & $0.0673(13)$ & $0.0396(9)$ & $0.0538(11)$ & $-0.0183(9)$ & $-0.0182(10)$ & $0.0129(8)$ \\
C9 & $0.1052(17)$ & $0.0496(11)$ & $0.0515(12)$ & $-0.0336(11)$ & $-0.0238(12)$ & $0.0084(9)$ \\
C10 & $0.0373(9)$ & $0.0488(10)$ & $0.0457(10)$ & $-0.0207(8)$ & $-0.0123(7)$ & $0.0004(8)$ \\
C11 & $0.0381(9)$ & $0.0392(8)$ & $0.0378(9)$ & $-0.0160(7)$ & $-0.0143(7)$ & $0.0012(7)$ \\
C12 & $0.0386(9)$ & $0.0445(9)$ & $0.0524(11)$ & $-0.0217(8)$ & $-0.0161(8)$ & $0.0050(8)$ \\
C13 & $0.0387(9)$ & $0.0364(8)$ & $0.0409(9)$ & $-0.0130(7)$ & $-0.0121(7)$ & $0.0020(7)$ \\
C14 & $0.0491(11)$ & $0.0502(10)$ & $0.0512(11)$ & $-0.0204(9)$ & $-0.0100(9)$ & $0.0125(8)$ \\
C15 & $0.0673(13)$ & $0.0505(11)$ & $0.0427(11)$ & $-0.0132(10)$ & $-0.0155(9)$ & $0.0126(8)$ \\
C16 & $0.0619(12)$ & $0.0492(10)$ & $0.0451(10)$ & $-0.0112(9)$ & $-0.0272(9)$ & $0.0035(8)$ \\
C17 & $0.0461(10)$ & $0.0461(10)$ & $0.0478(10)$ & $-0.0165(8)$ & $-0.0218(8)$ & $0.0029(8)$ \\
C18 & $0.0375(9)$ & $0.0334(8)$ & $0.0364(9)$ & $-0.0109(7)$ & $-0.0139(7)$ & $0.0002(6)$ \\
N1 & $0.0368(7)$ & $0.0413(7)$ & $0.0367(7)$ & $-0.0192(6)$ & $-0.0152(6)$ & $0.0041(6)$ \\
N2 & $0.0429(8)$ & $0.0546(8)$ & $0.0423(8)$ & $-0.0257(7)$ & $-0.0232(6)$ & $0.0079(7)$ \\
O1 & $0.0582(8)$ & $0.0530(7)$ & $0.0509(7)$ & $-0.0340(6)$ & $-0.0203(6)$ & $0.0023(6)$ \\
O2 & $0.0567(8)$ & $0.0639(8)$ & $0.0401(7)$ & $-0.0323(6)$ & $-0.0222(6)$ & $0.0146(6)$ \\
O3 & $0.0605(8)$ & $0.0829(10)$ & $0.0790(10)$ & $-0.0490(8)$ & $-0.0326(7)$ & $0.0265(8)$ \\
& & & & & & \\
\hline & & & & & &
\end{tabular}

Geometric parameters $\left(\AA,{ }^{\circ}\right)$

\begin{tabular}{llll}
\hline $\mathrm{C} 1-\mathrm{O} 1$ & $1.443(2)$ & $\mathrm{C} 10-\mathrm{N} 1$ & $1.4641(19)$ \\
$\mathrm{C} 1-\mathrm{C} 9$ & $1.525(3)$ & $\mathrm{C} 10-\mathrm{H} 10 \mathrm{~A}$ & 0.9700 \\
$\mathrm{C} 1-\mathrm{C} 2$ & $1.525(3)$ & $\mathrm{C} 10-\mathrm{H} 10 \mathrm{~B}$ & 0.9700 \\
$\mathrm{C} 1-\mathrm{H} 1$ & 0.9800 & $\mathrm{C} 11-\mathrm{O} 2$ & $1.2247(19)$ \\
$\mathrm{C} 2-\mathrm{C} 3$ & $1.499(3)$ & $\mathrm{C} 11-\mathrm{N} 2$ & $1.3663(19)$ \\
$\mathrm{C} 2-\mathrm{H} 2 \mathrm{~A}$ & 0.9700 & $\mathrm{C} 11-\mathrm{N} 1$ & $1.3725(19)$ \\
$\mathrm{C} 2-\mathrm{H} 2 \mathrm{~B}$ & 0.9700 & $\mathrm{C} 12-\mathrm{O} 3$ & $1.2144(18)$ \\
$\mathrm{C} 3-\mathrm{C} 4$ & $1.378(3)$ & $\mathrm{C} 12-\mathrm{N} 2$ & $1.375(2)$ \\
$\mathrm{C} 3-\mathrm{C} 8$ & $1.382(3)$ & $\mathrm{C} 12-\mathrm{C} 13$ & $1.460(2)$
\end{tabular}




\begin{tabular}{|c|c|c|c|}
\hline $\mathrm{C} 4-\mathrm{C} 5$ & $1.372(3)$ & $\mathrm{C} 13-\mathrm{C} 18$ & $1.389(2)$ \\
\hline $\mathrm{C} 4-\mathrm{H} 4$ & 0.9300 & $\mathrm{C} 13-\mathrm{C} 14$ & $1.393(2)$ \\
\hline $\mathrm{C} 5-\mathrm{C} 6$ & $1.362(3)$ & $\mathrm{C} 14-\mathrm{C} 15$ & $1.370(3)$ \\
\hline $\mathrm{C} 5-\mathrm{H} 5$ & 0.9300 & $\mathrm{C} 14-\mathrm{H} 14$ & 0.9300 \\
\hline $\mathrm{C} 6-\mathrm{C} 7$ & $1.377(3)$ & $\mathrm{C} 15-\mathrm{C} 16$ & $1.378(3)$ \\
\hline $\mathrm{C} 6-\mathrm{H} 6$ & 0.9300 & $\mathrm{C} 15-\mathrm{H} 15$ & 0.9300 \\
\hline $\mathrm{C} 7-\mathrm{C} 8$ & $1.383(3)$ & $\mathrm{C} 16-\mathrm{C} 17$ & $1.371(2)$ \\
\hline $\mathrm{C} 7-\mathrm{H} 7$ & 0.9300 & $\mathrm{C} 16-\mathrm{H} 16$ & 0.9300 \\
\hline $\mathrm{C} 8-\mathrm{C} 9$ & $1.495(3)$ & $\mathrm{C} 17-\mathrm{C} 18$ & $1.394(2)$ \\
\hline C9-H9A & 0.9700 & $\mathrm{C} 17-\mathrm{H} 17$ & 0.9300 \\
\hline С9-H9B & 0.9700 & $\mathrm{C} 18-\mathrm{N} 1$ & $1.410(2)$ \\
\hline $\mathrm{C} 10-\mathrm{O} 1$ & $1.4011(19)$ & $\mathrm{N} 2-\mathrm{H} 2$ & 0.8600 \\
\hline $\mathrm{O} 1-\mathrm{C} 1-\mathrm{C} 9$ & $110.81(16)$ & $\mathrm{O} 1-\mathrm{C} 10-\mathrm{H} 10 \mathrm{~A}$ & 109.1 \\
\hline $\mathrm{O} 1-\mathrm{C} 1-\mathrm{C} 2$ & $106.40(17)$ & $\mathrm{N} 1-\mathrm{C} 10-\mathrm{H} 10 \mathrm{~A}$ & 109.1 \\
\hline $\mathrm{C} 9-\mathrm{C} 1-\mathrm{C} 2$ & $105.33(16)$ & $\mathrm{O} 1-\mathrm{C} 10-\mathrm{H} 10 \mathrm{~B}$ & 109.1 \\
\hline $\mathrm{O} 1-\mathrm{C} 1-\mathrm{H} 1$ & 111.3 & $\mathrm{~N} 1-\mathrm{C} 10-\mathrm{H} 10 \mathrm{~B}$ & 109.1 \\
\hline $\mathrm{C} 9-\mathrm{C} 1-\mathrm{H} 1$ & 111.3 & $\mathrm{H} 10 \mathrm{~A}-\mathrm{C} 10-\mathrm{H} 10 \mathrm{~B}$ & 107.8 \\
\hline $\mathrm{C} 2-\mathrm{C} 1-\mathrm{H} 1$ & 111.3 & $\mathrm{O} 2-\mathrm{C} 11-\mathrm{N} 2$ & $121.04(15)$ \\
\hline $\mathrm{C} 3-\mathrm{C} 2-\mathrm{C} 1$ & $104.25(17)$ & $\mathrm{O} 2-\mathrm{C} 11-\mathrm{N} 1$ & $122.58(14)$ \\
\hline $\mathrm{C} 3-\mathrm{C} 2-\mathrm{H} 2 \mathrm{~A}$ & 110.9 & $\mathrm{~N} 2-\mathrm{C} 11-\mathrm{N} 1$ & $116.37(14)$ \\
\hline $\mathrm{C} 1-\mathrm{C} 2-\mathrm{H} 2 \mathrm{~A}$ & 110.9 & $\mathrm{O} 3-\mathrm{C} 12-\mathrm{N} 2$ & $120.33(16)$ \\
\hline $\mathrm{C} 3-\mathrm{C} 2-\mathrm{H} 2 \mathrm{~B}$ & 110.9 & $\mathrm{O} 3-\mathrm{C} 12-\mathrm{C} 13$ & $124.94(16)$ \\
\hline $\mathrm{C} 1-\mathrm{C} 2-\mathrm{H} 2 \mathrm{~B}$ & 110.9 & $\mathrm{~N} 2-\mathrm{C} 12-\mathrm{C} 13$ & $114.72(13)$ \\
\hline $\mathrm{H} 2 \mathrm{~A}-\mathrm{C} 2-\mathrm{H} 2 \mathrm{~B}$ & 108.9 & $\mathrm{C} 18-\mathrm{C} 13-\mathrm{C} 14$ & $119.96(16)$ \\
\hline $\mathrm{C} 4-\mathrm{C} 3-\mathrm{C} 8$ & $120.32(18)$ & $\mathrm{C} 18-\mathrm{C} 13-\mathrm{C} 12$ & $119.93(15)$ \\
\hline $\mathrm{C} 4-\mathrm{C} 3-\mathrm{C} 2$ & $129.21(19)$ & $\mathrm{C} 14-\mathrm{C} 13-\mathrm{C} 12$ & $120.11(15)$ \\
\hline $\mathrm{C} 8-\mathrm{C} 3-\mathrm{C} 2$ & $110.47(17)$ & $\mathrm{C} 15-\mathrm{C} 14-\mathrm{C} 13$ & $120.45(17)$ \\
\hline $\mathrm{C} 5-\mathrm{C} 4-\mathrm{C} 3$ & $119.3(2)$ & $\mathrm{C} 15-\mathrm{C} 14-\mathrm{H} 14$ & 119.8 \\
\hline $\mathrm{C} 5-\mathrm{C} 4-\mathrm{H} 4$ & 120.4 & $\mathrm{C} 13-\mathrm{C} 14-\mathrm{H} 14$ & 119.8 \\
\hline $\mathrm{C} 3-\mathrm{C} 4-\mathrm{H} 4$ & 120.4 & $\mathrm{C} 14-\mathrm{C} 15-\mathrm{C} 16$ & $119.26(17)$ \\
\hline $\mathrm{C} 6-\mathrm{C} 5-\mathrm{C} 4$ & $120.5(2)$ & $\mathrm{C} 14-\mathrm{C} 15-\mathrm{H} 15$ & 120.4 \\
\hline $\mathrm{C} 6-\mathrm{C} 5-\mathrm{H} 5$ & 119.8 & $\mathrm{C} 16-\mathrm{C} 15-\mathrm{H} 15$ & 120.4 \\
\hline $\mathrm{C} 4-\mathrm{C} 5-\mathrm{H} 5$ & 119.8 & $\mathrm{C} 17-\mathrm{C} 16-\mathrm{C} 15$ & $121.49(18)$ \\
\hline $\mathrm{C} 5-\mathrm{C} 6-\mathrm{C} 7$ & $121.1(2)$ & $\mathrm{C} 17-\mathrm{C} 16-\mathrm{H} 16$ & 119.3 \\
\hline $\mathrm{C} 5-\mathrm{C} 6-\mathrm{H} 6$ & 119.4 & $\mathrm{C} 15-\mathrm{C} 16-\mathrm{H} 16$ & 119.3 \\
\hline $\mathrm{C} 7-\mathrm{C} 6-\mathrm{H} 6$ & 119.4 & $\mathrm{C} 16-\mathrm{C} 17-\mathrm{C} 18$ & $119.68(16)$ \\
\hline $\mathrm{C} 6-\mathrm{C} 7-\mathrm{C} 8$ & $118.7(2)$ & $\mathrm{C} 16-\mathrm{C} 17-\mathrm{H} 17$ & 120.2 \\
\hline $\mathrm{C} 6-\mathrm{C} 7-\mathrm{H} 7$ & 120.6 & $\mathrm{C} 18-\mathrm{C} 17-\mathrm{H} 17$ & 120.2 \\
\hline $\mathrm{C} 8-\mathrm{C} 7-\mathrm{H} 7$ & 120.6 & $\mathrm{C} 13-\mathrm{C} 18-\mathrm{C} 17$ & $119.16(15)$ \\
\hline $\mathrm{C} 3-\mathrm{C} 8-\mathrm{C} 7$ & 120.04 (19) & $\mathrm{C} 13-\mathrm{C} 18-\mathrm{N} 1$ & $119.53(14)$ \\
\hline $\mathrm{C} 3-\mathrm{C} 8-\mathrm{C} 9$ & $110.30(17)$ & $\mathrm{C} 17-\mathrm{C} 18-\mathrm{N} 1$ & $121.31(14)$ \\
\hline $\mathrm{C} 7-\mathrm{C} 8-\mathrm{C} 9$ & $129.6(2)$ & $\mathrm{C} 11-\mathrm{N} 1-\mathrm{C} 18$ & $122.06(13)$ \\
\hline $\mathrm{C} 8-\mathrm{C} 9-\mathrm{C} 1$ & $104.28(16)$ & $\mathrm{C} 11-\mathrm{N} 1-\mathrm{C} 10$ & $117.92(13)$ \\
\hline $\mathrm{C} 8-\mathrm{C} 9-\mathrm{H} 9 \mathrm{~A}$ & 110.9 & $\mathrm{C} 18-\mathrm{N} 1-\mathrm{C} 10$ & $119.96(13)$ \\
\hline $\mathrm{C} 1-\mathrm{C} 9-\mathrm{H} 9 \mathrm{~A}$ & 110.9 & $\mathrm{C} 11-\mathrm{N} 2-\mathrm{C} 12$ & $127.31(14)$ \\
\hline $\mathrm{C} 8-\mathrm{C} 9-\mathrm{H} 9 \mathrm{~B}$ & 110.9 & $\mathrm{C} 11-\mathrm{N} 2-\mathrm{H} 2$ & 116.3 \\
\hline $\mathrm{C} 1-\mathrm{C} 9-\mathrm{H} 9 \mathrm{~B}$ & 110.9 & $\mathrm{C} 12-\mathrm{N} 2-\mathrm{H} 2$ & 116.3 \\
\hline
\end{tabular}




\begin{tabular}{|c|c|c|c|}
\hline $\mathrm{H} 9 \mathrm{~A}-\mathrm{C} 9-\mathrm{H} 9 \mathrm{~B}$ & 108.9 & $\mathrm{C} 10-\mathrm{O} 1-\mathrm{C} 1$ & $114.24(13)$ \\
\hline $\mathrm{O} 1-\mathrm{C} 10-\mathrm{N} 1$ & $112.53(13)$ & & \\
\hline $\mathrm{O} 1-\mathrm{C} 1-\mathrm{C} 2-\mathrm{C} 3$ & $96.00(19)$ & $\mathrm{C} 14-\mathrm{C} 15-\mathrm{C} 16-\mathrm{C} 17$ & $-0.5(3)$ \\
\hline $\mathrm{C} 9-\mathrm{C} 1-\mathrm{C} 2-\mathrm{C} 3$ & $-21.7(2)$ & $\mathrm{C} 15-\mathrm{C} 16-\mathrm{C} 17-\mathrm{C} 18$ & $-0.2(3)$ \\
\hline $\mathrm{C} 1-\mathrm{C} 2-\mathrm{C} 3-\mathrm{C} 4$ & $-167.6(2)$ & $\mathrm{C} 14-\mathrm{C} 13-\mathrm{C} 18-\mathrm{C} 17$ & $-0.2(2)$ \\
\hline $\mathrm{C} 1-\mathrm{C} 2-\mathrm{C} 3-\mathrm{C} 8$ & $12.9(2)$ & $\mathrm{C} 12-\mathrm{C} 13-\mathrm{C} 18-\mathrm{C} 17$ & $179.10(15)$ \\
\hline $\mathrm{C} 8-\mathrm{C} 3-\mathrm{C} 4-\mathrm{C} 5$ & $0.2(3)$ & $\mathrm{C} 14-\mathrm{C} 13-\mathrm{C} 18-\mathrm{N} 1$ & $180.00(15)$ \\
\hline $\mathrm{C} 2-\mathrm{C} 3-\mathrm{C} 4-\mathrm{C} 5$ & $-179.3(2)$ & $\mathrm{C} 12-\mathrm{C} 13-\mathrm{C} 18-\mathrm{N} 1$ & $-0.7(2)$ \\
\hline $\mathrm{C} 3-\mathrm{C} 4-\mathrm{C} 5-\mathrm{C} 6$ & $-0.9(3)$ & $\mathrm{C} 16-\mathrm{C} 17-\mathrm{C} 18-\mathrm{C} 13$ & $0.6(2)$ \\
\hline $\mathrm{C} 4-\mathrm{C} 5-\mathrm{C} 6-\mathrm{C} 7$ & $0.4(3)$ & $\mathrm{C} 16-\mathrm{C} 17-\mathrm{C} 18-\mathrm{N} 1$ & $-179.66(15)$ \\
\hline $\mathrm{C} 5-\mathrm{C} 6-\mathrm{C} 7-\mathrm{C} 8$ & $0.8(3)$ & $\mathrm{O} 2-\mathrm{C} 11-\mathrm{N} 1-\mathrm{C} 18$ & $177.94(15)$ \\
\hline $\mathrm{C} 4-\mathrm{C} 3-\mathrm{C} 8-\mathrm{C} 7$ & $1.0(3)$ & $\mathrm{N} 2-\mathrm{C} 11-\mathrm{N} 1-\mathrm{C} 18$ & $-3.1(2)$ \\
\hline $\mathrm{C} 2-\mathrm{C} 3-\mathrm{C} 8-\mathrm{C} 7$ & $-179.43(19)$ & $\mathrm{O} 2-\mathrm{C} 11-\mathrm{N} 1-\mathrm{C} 10$ & $-4.9(2)$ \\
\hline $\mathrm{C} 4-\mathrm{C} 3-\mathrm{C} 8-\mathrm{C} 9$ & $-178.03(17)$ & $\mathrm{N} 2-\mathrm{C} 11-\mathrm{N} 1-\mathrm{C} 10$ & $174.07(14)$ \\
\hline $\mathrm{C} 2-\mathrm{C} 3-\mathrm{C} 8-\mathrm{C} 9$ & $1.6(2)$ & $\mathrm{C} 13-\mathrm{C} 18-\mathrm{N} 1-\mathrm{C} 11$ & $2.8(2)$ \\
\hline $\mathrm{C} 6-\mathrm{C} 7-\mathrm{C} 8-\mathrm{C} 3$ & $-1.4(3)$ & $\mathrm{C} 17-\mathrm{C} 18-\mathrm{N} 1-\mathrm{C} 11$ & $-176.99(15)$ \\
\hline $\mathrm{C} 6-\mathrm{C} 7-\mathrm{C} 8-\mathrm{C} 9$ & $177.3(2)$ & $\mathrm{C} 13-\mathrm{C} 18-\mathrm{N} 1-\mathrm{C} 10$ & $-174.32(14)$ \\
\hline $\mathrm{C} 3-\mathrm{C} 8-\mathrm{C} 9-\mathrm{C} 1$ & $-15.3(2)$ & $\mathrm{C} 17-\mathrm{C} 18-\mathrm{N} 1-\mathrm{C} 10$ & $5.9(2)$ \\
\hline $\mathrm{C} 7-\mathrm{C} 8-\mathrm{C} 9-\mathrm{C} 1$ & $165.8(2)$ & $\mathrm{O} 1-\mathrm{C} 10-\mathrm{N} 1-\mathrm{C} 11$ & $103.02(16)$ \\
\hline $\mathrm{O} 1-\mathrm{C} 1-\mathrm{C} 9-\mathrm{C} 8$ & $-92.08(18)$ & $\mathrm{O} 1-\mathrm{C} 10-\mathrm{N} 1-\mathrm{C} 18$ & $-79.75(18)$ \\
\hline $\mathrm{C} 2-\mathrm{C} 1-\mathrm{C} 9-\mathrm{C} 8$ & $22.6(2)$ & $\mathrm{O} 2-\mathrm{C} 11-\mathrm{N} 2-\mathrm{C} 12$ & $-179.57(16)$ \\
\hline $\mathrm{O} 3-\mathrm{C} 12-\mathrm{C} 13-\mathrm{C} 18$ & $179.98(17)$ & $\mathrm{N} 1-\mathrm{C} 11-\mathrm{N} 2-\mathrm{C} 12$ & $1.4(2)$ \\
\hline $\mathrm{N} 2-\mathrm{C} 12-\mathrm{C} 13-\mathrm{C} 18$ & $-0.9(2)$ & $\mathrm{O} 3-\mathrm{C} 12-\mathrm{N} 2-\mathrm{C} 11$ & $179.69(17)$ \\
\hline $\mathrm{O} 3-\mathrm{C} 12-\mathrm{C} 13-\mathrm{C} 14$ & $-0.7(3)$ & $\mathrm{C} 13-\mathrm{C} 12-\mathrm{N} 2-\mathrm{C} 11$ & $0.5(2)$ \\
\hline $\mathrm{N} 2-\mathrm{C} 12-\mathrm{C} 13-\mathrm{C} 14$ & $178.46(16)$ & $\mathrm{N} 1-\mathrm{C} 10-\mathrm{O} 1-\mathrm{C} 1$ & $-93.88(17)$ \\
\hline $\mathrm{C} 18-\mathrm{C} 13-\mathrm{C} 14-\mathrm{C} 15$ & $-0.5(3)$ & $\mathrm{C} 9-\mathrm{C} 1-\mathrm{O} 1-\mathrm{C} 10$ & $-73.73(18)$ \\
\hline $\mathrm{C} 12-\mathrm{C} 13-\mathrm{C} 14-\mathrm{C} 15$ & $-179.80(17)$ & $\mathrm{C} 2-\mathrm{C} 1-\mathrm{O} 1-\mathrm{C} 10$ & $172.28(14)$ \\
\hline $\mathrm{C} 13-\mathrm{C} 14-\mathrm{C} 15-\mathrm{C} 16$ & $0.8(3)$ & & \\
\hline
\end{tabular}

Hydrogen-bond geometry $\left(\AA,{ }^{\circ}\right)$

\begin{tabular}{lllll}
\hline$D-\mathrm{H} \cdots A$ & $D-\mathrm{H}$ & $\mathrm{H} \cdots A$ & $D \cdots A$ & $D-\mathrm{H}^{\cdots} \cdots A$ \\
\hline $\mathrm{N} 2-\mathrm{H} 2 \cdots \mathrm{O}^{2}$ & 0.86 & 2.06 & $2.9106(18)$ & 169 \\
$\mathrm{C} 9-\mathrm{H} 9 A \cdots \mathrm{O} 2^{\mathrm{ii}}$ & 0.97 & 2.56 & $3.527(3)$ & 173 \\
$\mathrm{C} 16-\mathrm{H} 16 \cdots{ }^{\mathrm{iii}}$ & 0.93 & 2.47 & $3.378(2)$ & 166 \\
$\mathrm{C} 10-\mathrm{H} 10 A \cdots{ }^{\mathrm{iii}}{ }^{\text {iv }}$ & 0.97 & 2.46 & $3.404(2)$ & 165 \\
$\mathrm{C} 5-\mathrm{H} 5 \cdots \mathrm{O}^{v}$ & 0.93 & 2.47 & $3.314(2)$ & 151 \\
\hline
\end{tabular}

Symmetry codes: (i) $-x,-y+1,-z$; (ii) $-x+1,-y+1,-z$; (iii) $-x+1,-y+1,-z+1$; (iv) $x+1, y, z$; (v) $x+1, y+1, z$. 